\title{
Approaches of combining a 3D-printed elastic structure and a hydrogel to create models for plant-inspired actuators
}

\author{
Nadia Rodriguez ${ }^{1} \cdot$ Anil K. Bastola $^{1} \cdot$ Marc Behl $^{1} \cdot$ Patricia Soffiatti $^{3} \cdot$ Nick P. Rowe $^{4} \cdot$ Andreas Lendlein $^{1,2}$ (D)
}

Received: 13 April 2021 / Accepted: 7 June 2021 / Published online: 28 June 2021

(c) Helmholtz-Zentrum Hereon 2021

\begin{abstract}
Inspired by the interesting functional traits of a climbing cactus, Selenicereus setaceus, found in the forest formations of Southeastern Brazil, we formulated a hypothesis that we can directly learn from the plants to develop multi-functional artificial systems by means of a multi-disciplinary approach. In this context, our approach is to take advantage of 3D-printing techniques and shape-memory hydrogels synergistically to mimic the functional traits of the cactus. This work reports on the preliminary investigation of cactus-inspired artificial systems. First, we 3D-printed soft polymeric materials and characterized them, which defines the structure and is a passive component of a multi-material system. Second, different hydrogels were synthesized and characterized, which is an active component of a multi-material system. Finally, we investigated how the hydrogel can be integrated into the 3D-printed constructs to develop artificial functional systems.
\end{abstract}

\section{Introduction}

A number of fascinating activities exhibited by plants in nature by virtue of growth are a wonderful source of inspiration to develop new technologies. The evolutionary history and growth of plants lead to functional traits that can anchor, twine, vine, or search. An interesting representative in this context is Selenicereus setaceus, a cactus found in the dry forest formations of Southeastern Brazil, capable of climbing [1]. Such biological functional systems can serve as a source for bio-inspired robots with the potential ability to anchor, attach, and climb [2,3]. We can question how the process of such developments in nature could be represented as technical artefacts. One of the potential approaches is the evolutionary design of a multi-material system inspired by the plants behaviours. Selenicereus setaceus demonstrates a

Andreas Lendlein

andreas.lendlein@hereon.de

1 Institute of Active Polymers, Helmholtz-Zentrum Hereon, Kantstr. 55, 14513 Teltow, Germany

2 Berlin-Brandenburg Center for Regenerative Therapies, Helmholtz-Zentrum Hereon, Kantstr. 55, 14513 Teltow, Germany

3 Department of Botany, Federal University of Parana State, Curitiba, PR, Brazil

4 AMAP, Univ Montpellier, CIRAD, CNRS, INRAE, IRD, Montpellier, France unique structural configuration along the different stages of growth: the stem is circular at the base, while the younger parts are star-like in shape at the apex. The cactus consists of soft tissues surrounded by a thin skin layer (Fig. 1). The capability of transformation in shape optimizes its flexural rigidity and also provides flexibility to allow the cactus to search for light in the challenging environment of the seasonally dry Atlantic forest [1]. The mechanical performance and various interesting functional traits of the cactus have been extensively explored in previous studies [1, 4].

A hydrogel is a polymer network, which is crosslinked and able to swell in aqueous media caused by its hydrophilic components. Hydrogels are materials displaying macroscale movements from non-directional swelling to unidirectional movement as presented by shape-memory function [5-8]. Besides, hydrogels are capable of water soaking and water storage [6-10]. Hydrogels are, therefore, one of the promising candidates to mimic the climbing movement of living plants such as Selenicereus setaceus.

Herein, we explore how to mimic the interesting functional traits of the cactus by combining a digital manufacturing technique and shape-memory effect (SME) in hydrogels towards the plant-inspired robotic systems. We consider several aspects of this highly promising cactus species. Selenicereus setaceus offers such a wide range of cross-sectional shapes, and it, therefore, can be mimicked by the swelling behaviour of hydrogels. Similarly, the stem of the cactus is able to deform and twist with limited benign failure when 
Fig. 1 (a) Photographs of Selenicereus setaceus: showing climbing, searching, and different cross sections at different stages of growth. Conceptual design to mimic the shape (b) and to mimic the movements (c) of the cactus
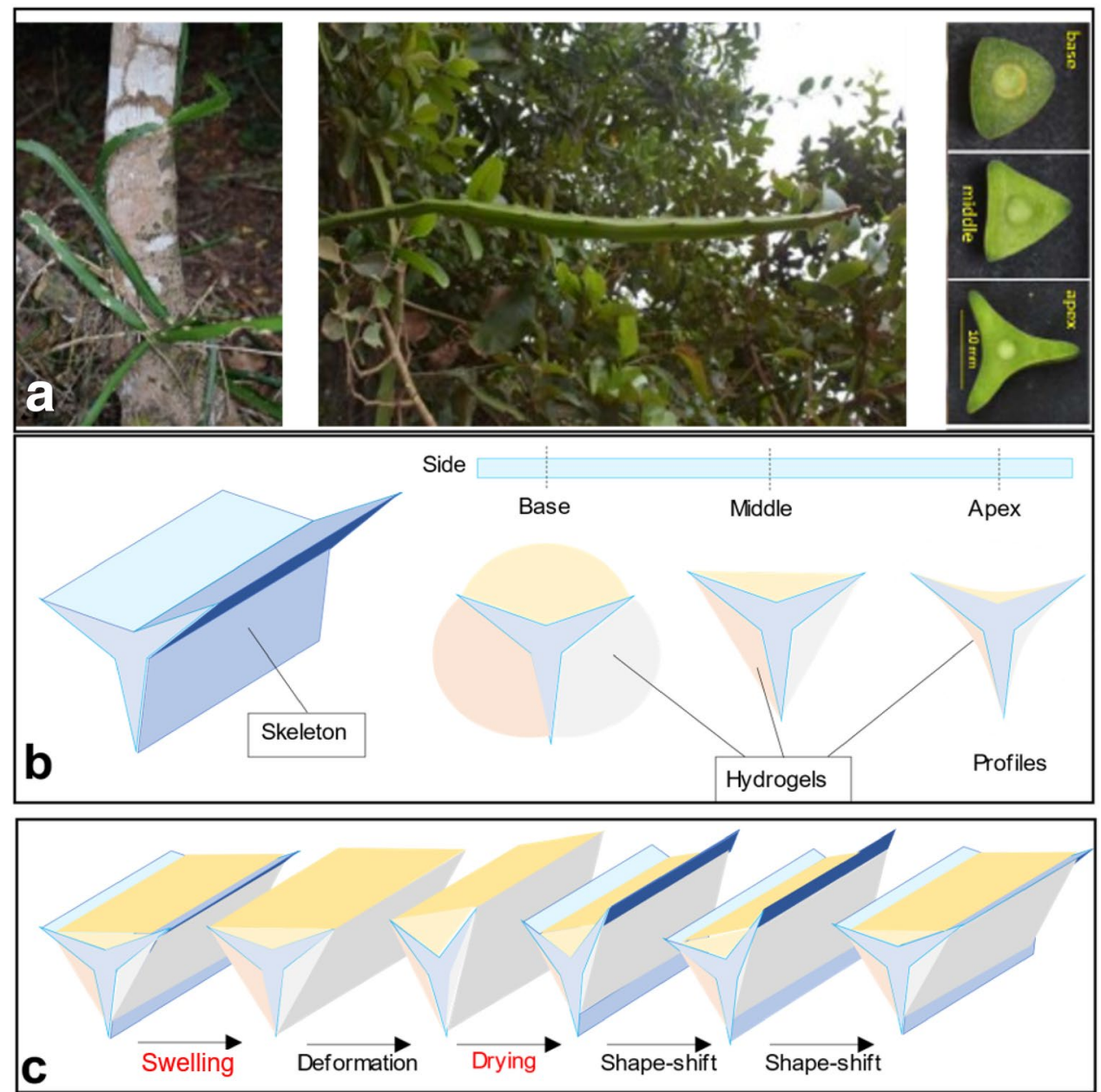

physical conditions force it to slip from the supporting vegetation, as well as climb up and out of the dark understorey vegetation. Plant growth is also related to the amount of water found in the surrounding environment. Climbing of the cactus depends on attachment points while apex growth is directed towards the light. Therefore, swelling behaviour and SME of hydrogels can be a useful tool to mimic the twisting and growing movements of the cactus. Furthermore, the overall shape of the cactus is complex and specific. In order to create such highly tailored shapes, a number of advantages are offered by additive manufacturing (AM). AM or $3 \mathrm{D}$-printing is a digital manufacturing process and can create highly complex geometries in a layer-by-layer fashion [11-14]. It is undoubtedly a suitable technique to realize the complex geometries offered by plants.

\section{Hypothesis}

A solid star-shape profile will be 3D-printed and used as a skeleton to shape the final object to match the geometry of the cactus. The skeleton material must also be deformable in order to undergo different kinds of movements. Each outer groove will be filled up with hydrogels based on the same or different compositions. By controlling the amount of water introduced into the polymer network along with the hydrogel, cross sections of the object will display similar profiles comparable with Selenicereus setaceus (Fig. 1b, c). Once the hydrogels are swollen, the multi-material system can be deformed (elongate, twist, bend, etc.). Following this, drying of the hydrogels results in the deformed shape being fixed. Hydrogels can be swollen again based on different swelling kinetics, different degree of swelling, or different amounts of water; the permanent shape can then be recovered in a stepwise manner (Fig. 1c). 


\section{Method}

Gel polymerization was performed according to reference [15]. PEG-DM was heated to $60{ }^{\circ} \mathrm{C}$. Then, the polymer containing either vinyl or methacrylate functions $(\mathrm{X})$ and afterwards $1.5 \mathrm{~mol} \%$ of 2,2'-azobis(2-methyl-propionitile) (AIBN) (relative to poly(ethylene glycol) di-methacrylate (PEG-DM) and $\mathrm{X}$ ) were added and mixed until a homogeneous mixture was obtained (supplement data Table S1). The hydrogel solution was then poured into a mould. Crosslinking of the gel was performed without stirring at $75{ }^{\circ} \mathrm{C}$ for $24 \mathrm{~h}$. Further details of materials and methods can be found in the Supporting information.

\section{Results and discussion}

\section{D-printing}

Skeletons were created using Agilus30 (AG) and TangoBlackPlus (TBP) as model materials for the 3D-printer. Both materials were selected because, according to the datasheet provided by Stratasys, they have the highest elongation at break $\left(\varepsilon_{\mathrm{b}}\right)$ values [16]. Furthermore, both substances provide low $T_{\mathrm{g}}, 2 \pm 2{ }^{\circ} \mathrm{C}$ in case of $\mathrm{AG}$, and $-4 \pm 2{ }^{\circ} \mathrm{C}$ in case of TBP (Supporting Information, Fig. S1). In addition, both substances provide high thermal stability as shown for AG, which displays a first decrease of mass above $230{ }^{\circ} \mathrm{C}$ to around $50 \%$ at $330{ }^{\circ} \mathrm{C}$ and a second decrease above $380{ }^{\circ} \mathrm{C}$ to $10 \%$ at around $460{ }^{\circ} \mathrm{C}$ (Fig. S2).

The major dimensions of the skeleton are length $(L)$, diameter $(D)$, and width between the centre point and groove $(W)$ (see Fig. 2a), which defines the star-shape similar to the cactus. In order to perform moulding of hydrogels, the skeleton was printed with closed extremities (see Fig. 2b).

As the printed material should be used as a mould for hydrogel synthesis, the skeleton material was investigated for its compatibility with hydrogel synthesis conditions. As shown in Table 1, both AG and TBP were immersed in various solvents and hydrogel components. It is found that printed materials swell in organic solvents, for example, up to $200 \mathrm{wt} \%$ in polar solvents like ethanol and up to $500 \mathrm{wt} \%$ in non-polar solvents like chloroform; however, an influence of temperature (RT or $60{ }^{\circ} \mathrm{C}$ ) is not observed. Interestingly, the printed materials exhibit non-swelling behaviour in water, which, therefore, can be a suitable solvent for hydrogel synthesis. Likewise, printed materials do not swell in PEG-DM solution, which therefore can be a potential component of the hydrogel network. A difference of the swelling ability between the two model materials was not detected. These tests indicated that the use of organic solvents for polymer network synthesis should be performed carefully as mechanical properties of materials are changed via swelling and could affect the attachment of hydrogels onto the surface of the skeleton.

Furthermore, to mimic plant movements such as bending, twisting, and even growing, mechanical properties such as elastic modulus and elongation must also be understood. Tensile tests were conducted with respect to printed directions $(\mathrm{x}, \mathrm{y}$, and $\mathrm{z})$ to determine Young's moduli $\left(E_{\mathrm{x}, \mathrm{y}, \mathrm{z}}\right)$ and $\varepsilon_{\mathrm{bx}, \mathrm{y}, \mathrm{z}}$. The $E_{\mathrm{x}}$ and $E_{\mathrm{y}}$ values of $\mathrm{AG}$ and TBP were around $600 \mathrm{kPa}$ and $500 \mathrm{kPa}$, respectively, but $E_{\mathrm{z}}$ values of both materials were halved. The $\varepsilon_{\mathrm{bx}}$ and $\varepsilon_{\mathrm{by}}$ of AG were in accordance with values from Stratasys datasheet. However, $\varepsilon_{\mathrm{bz}}$ of $\mathrm{AG}$ is $15 \%$ lower than $\varepsilon_{\mathrm{bx}}$ and $\varepsilon_{\mathrm{by}}$. The $\varepsilon_{\mathrm{bx}}$ and $\varepsilon_{\mathrm{by}}$ of TBP were lower than values from the provider but $\varepsilon_{\mathrm{bz}}$ value was even lower, i.e. $30 \%$ lower (supplement data Table S2). Therefore, it should be noted that the printed materials have different mechanical properties depending on 3D-printed directions.

\section{Hydrogel synthesis and moulding on 3D-printed skeleton}

Several copolymer networks with different compositions were polymerized by free radical chain polymerization (FRCP) in presence of PEG-DM as crosslinker and AIBN as initiator. The NVP, DEG-VE, PEG-M, and PEG-MEM were used as hydrophilic monomers.

As found in immersion tests, the printed materials swell in organic solvents. Therefore, bulk polymerization had to be selected over solution polymerization. Gels based on NVP and $0.5 \mathrm{~mol} \%, 3 \mathrm{~mol} \%, 30 \mathrm{~mol} \%$, or $50 \mathrm{~mol} \%$ of crosslinker were obtained (see Fig. 2, panel b). The gel content ( $G$ values, see Supplement data for detail) equalled to $31 \pm 2 \%$, $30 \pm 2 \%, 81 \pm 2 \%$, and $85 \pm 5 \%$, respectively. Low $G$ values can be attributed to the low content of the crosslinker resulting in free backbone chains, which could be washed away during the extraction procedure. However, when the gel solution was moulded onto the skeleton surface, the hydrogel solution was immediately absorbed by the printed material (Supplement data Figure S3).

In order to prevent absorption of gel solution, new solutions having higher viscosity were prepared. Increased viscosity was obtained by using monomers with longer side chains as backbone units. The DEG-VE, PEG-M, and PEGMEM polymers were selected since their boiling point $\left(b_{\mathrm{p}}\right)$ and melting point $\left(T_{\mathrm{m}}\right)$ are suitable with reaction conditions $\left(b_{\mathrm{p} \mathrm{DEG}-\mathrm{VE}}=196^{\circ} \mathrm{C}, b_{\mathrm{p} \text { PEG-M }}>75^{\circ} \mathrm{C}, T_{\mathrm{m} \text {,PEG-MEM }} \approx 40^{\circ} \mathrm{C}\right.$ ). Gels were successfully synthesized. The detail of the gel`s $\mathrm{G}$ values, mass swelling degree, and swelling kinetics are given in supplement data (Table S3 and Figure S4). However, again during moulding, the solutions were still absorbed and filled material pores and interlayer spaces. The absorption of gel solutions in between layers of the printed objects 
Fig. 2 (a) CAD model of the star-shaped skeleton and 3D-printed sample. (b) Structures of synthesized polymer hydrogel networks; network containing NVP and PEG-DM (i), DEG-VE and PEG-DM (ii), PEG-ME and PEG-DM (iii), PEG-MEM and PEG-DM (iv); PEG-DM was a crosslinker. (c) Moulding of strategies of hydrogel solution on 3D-printed skeleton: pre-polymerization (1), soaking (2), and successive mouldings (3). Scale bar: $1 \mathrm{~cm}$
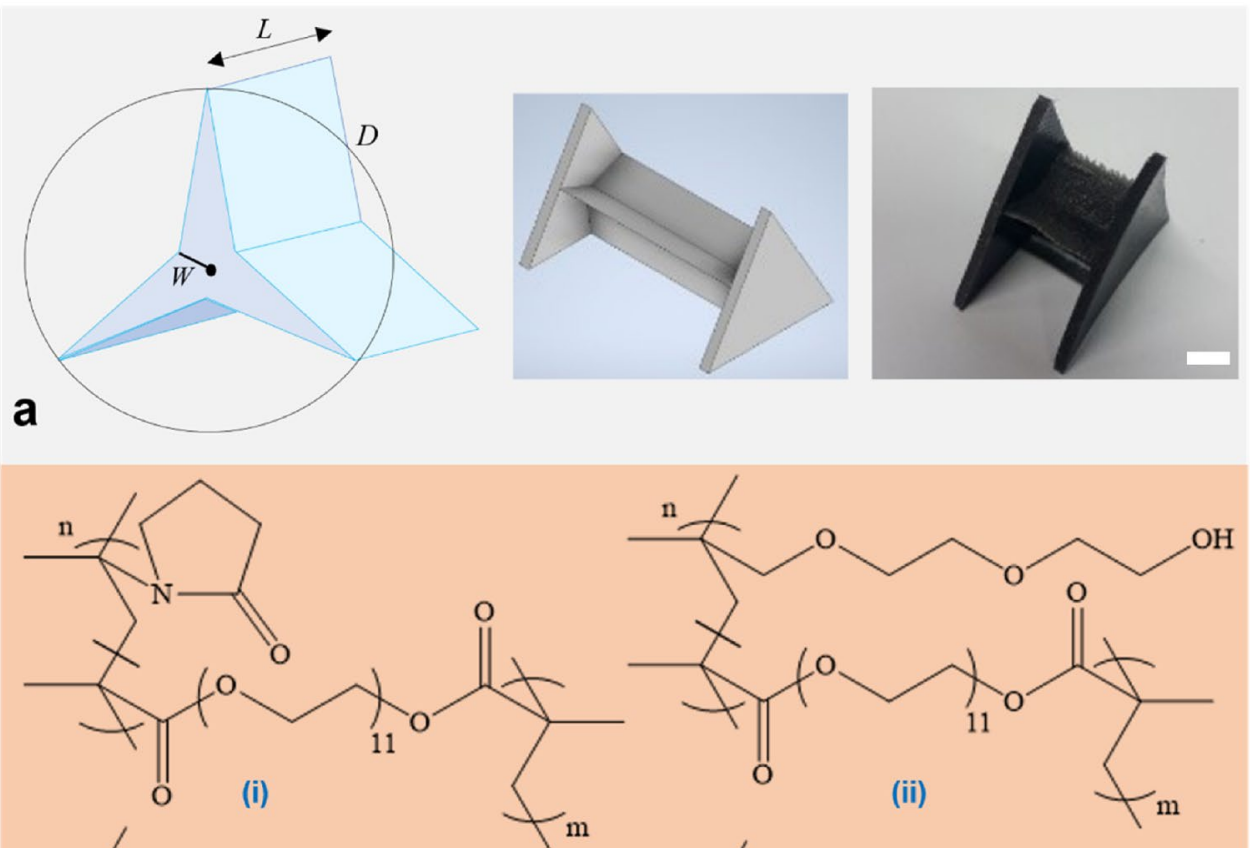

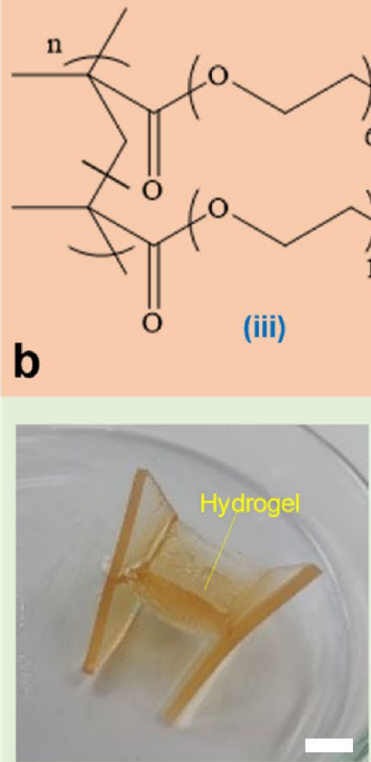

C (1)

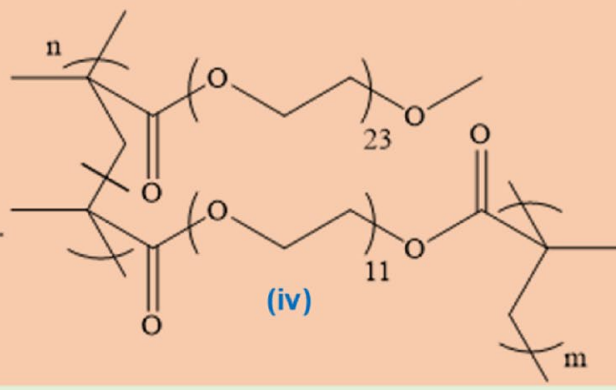

was confirmed by successful polymerization and swelling of hydrogel on an elastomer surface.

Lastly, three different alternative strategies were performed in order to mould hydrogels in the 3D-printed skeleton: (1) pre-polymerization, (2) soaking, and (3) successive moulding. At first, it was speculated that pre-polymerization increases the viscosity of the solution, which should avoid the diffusion of the oligomers into the 3D-printed skeleton. Second, the skeleton was immersed in an excess amount of gel solution, so the printed material reached equilibrium swelling. The remaining solution would then polymerize around the skeleton and could be tightly connected to the skeleton surfaces. This alternative implies that in the three outer grooves, the same hydrogel can be polymerized only. Finally, by successive mouldings, in a first step, one groove would be filled whereas the solution would fill the skeleton porosity as well. When this soaked solution has been polymerized, additionally the hydrogel solution could be polymerized in the mould provided by one groove. This third procedure can be repeated with different types of hydrogel solutions for the two other groves. The third strategy is comparatively time consuming compared with the others. As shown in Fig. 2c, all three strategies: pre-polymerization, immersion, and successive moulding were successful to fill the outer grooves with polymerized gels. However, when the hydrogels were swollen, it could be noted that the hydrogel 
Table 1 Degree of swelling $\left(Q_{\mathrm{w}, \text { print }}\right)$ and qualitative description of mechanical properties of printed AG and TBP materials at room temperature (RT) and $60^{\circ} \mathrm{C}$

\begin{tabular}{llll}
\hline $\begin{array}{l}\text { Swelling solvent/component of } \\
\text { hydrogel synthesis }\end{array}$ & Temperature & $Q_{\mathrm{w}, \text { print }}$ wt $\left.\%\right)$ & $\begin{array}{l}\text { Qualitative description } \\
\text { after immersion }\end{array}$ \\
\hline 1,2-Dichloroethane & $\mathrm{RT}$ & $400 \pm 100$ & Brittle \\
1,4-Dioxane & $\mathrm{RT}$ & $400 \pm 100$ & Brittle \\
Chloroform & $\mathrm{RT}$ & $500 \pm 100$ & Brittle \\
NVP & $\mathrm{RT}$ & $400 \pm 100$ & Brittle \\
Ethanol & $\mathrm{RT}$ & $200 \pm 100$ & Brittle \\
Water & $\mathrm{RT}$ & - & Surface bleaching, ductile \\
PEG-DM & $\mathrm{RT}$ & - & Ductile \\
1,2-Dichloroethane & $60{ }^{\circ} \mathrm{C}$ & $500 \pm 100$ & Brittle \\
1,4-Dioxane & $60^{\circ} \mathrm{C}$ & $450 \pm 100$ & Brittle \\
NVP & $60^{\circ} \mathrm{C}$ & $400 \pm 100$ & Brittle \\
Ethanol & $60^{\circ} \mathrm{C}$ & $200 \pm 100$ & Brittle \\
Water & $60^{\circ} \mathrm{C}$ & - & Surface bleaching, ductile \\
PEG-DM & $60^{\circ} \mathrm{C}$ & - & Ductile \\
\hline
\end{tabular}

lost connection to the 3D-printed structure, which can be attributed to a mismatch between the elastic properties of the soft hydrogel and the stiff 3D-printed scaffold. Therefore, further optimizations and investigations of compatibility between hydrogel and printed materials are essential for the successful development of cactus-inspired multi-materials systems.

\section{Conclusions}

AM was used to print a skeleton with the star-like shape of the cactus. The skeleton was expected to be covered by hydrogels. However, it was observed that the moulding of hydrogels onto the skeleton surface was rather challenging. One of the possible reasons was related to the printing process, the skeleton exhibited different mechanical properties along the $\mathrm{z}$-axis compared to the $\mathrm{x}$-axis and $\mathrm{y}$-axis. This variation was related to the different crosslinking density of the printed materials. Thus, the hydrogel solution was more likely to be absorbed by the printed material in between the layers. On the other hand, compatibility of the two different materials also plays a vital role to attach hydrogel to the skeleton, and therefore, studying the chemical properties of the printed materials or the surface modification of the printed skeleton is necessary.

Alternative moulding strategies as attempted here such as pre-polymerization could be used to overcome the absorption issue. Moreover, it was observed that the printed material did not swell in water. Therefore, polymerization in water-based systems could be a potential alternative. The synthesis should be adapted with hydrogel components (monomer, crosslinker, and initiator) that are water soluble. The functions of the cactus-inspired technical system are reported in our study [17].
Supplementary Information The online version contains supplementary material available at https://doi.org/10.1557/s43580-021-00081-6.

Acknowledgments This work was financially supported by the Helmholtz Association through programme-oriented funding and received funding from the European Union's Horizon 2020 research and innovation program under Grant Agreement No. 824074 (GrowBot).

Funding Open Access funding enabled and organized by Projekt DEAL.

Data availability The datasets generated during and/or analysed during the current study are available from the corresponding author on reasonable request.

\section{Declarations}

Conflict of interest The authors have no relevant financial or non-financial interests to disclose.

Open Access This article is licensed under a Creative Commons Attribution 4.0 International License, which permits use, sharing, adaptation, distribution and reproduction in any medium or format, as long as you give appropriate credit to the original author(s) and the source, provide a link to the Creative Commons licence, and indicate if changes were made. The images or other third party material in this article are included in the article's Creative Commons licence, unless indicated otherwise in a credit line to the material. If material is not included in the article's Creative Commons licence and your intended use is not permitted by statutory regulation or exceeds the permitted use, you will need to obtain permission directly from the copyright holder. To view a copy of this licence, visit http://creativecommons.org/licenses/by/4.0/.

\section{References}

1. P. Soffiatti, N.P. Rowe, Mechanical innovations of a climbing cactus: functional insights for a new generation of growing robots. Front. Rob. AI 7 (2020). 
2. B. Mazzolai, L. Beccai, V. Mattoli, Plants as model in biomimetics and biorobotics: new perspectives. Front. Bioeng. Biotechnol. 2, 2 (2014)

3. B. Mazzolai, Plant-Inspired Growing Robots, in Soft Robotics: Trends, Applications and Challenges (Springer, Berlin, 2017), p. 57

4. A.K. Bastola, P. Soffiatti, M. Behl, A. Lendlein, N.P. Rowe, Structural performance of a climbing cactus: making the most of softness. J. R. Soc. Interface 18, 20210040 (2021)

5. Q. Zhao, M. Behl, A. Lendlein, Shape-memory polymers with multiple transitions: complex actively moving polymers. Soft Matter 9, 1744 (2013)

6. Y. Osada, A. Matsuda, Shape memory in hydrogels. Nature 376, 219 (1995)

7. C. Löwenberg, M. Balk, C. Wischke, M. Behl, A. Lendlein, Shape-memory hydrogels: evolution of structural principles to enable shape switching of hydrophilic polymer networks. Acc. Chem. Res. 50, 723 (2017)

8. A. Lendlein, S. Kelch, Shape-memory polymers. Angew. Chem. Int. Ed. 41, 2034 (2002)

9. S. Liu, A.K. Bastola, L. Li, A 3D printable and mechanically robust hydrogel based on alginate and graphene oxide. ACS Appl. Mater. Interfaces. 9, 41473 (2017)
10. X. Liu, J. Liu, S. Lin, X. Zhao, Hydrogel machines. Mater. Today 36, $102(2020)$

11. X. Wang, M. Jiang, Z. Zhou, J. Gou, D. Hui, 3D printing of polymer matrix composites: a review and prospective. Composites B 110, 442 (2017)

12. A. Sadeghi, A. Mondini, B. Mazzolai, Toward self-growing soft robots inspired by plant roots and based on additive manufacturing technologies. Soft Rob. 4, 211 (2017)

13. A.S. Gladman, E.A. Matsumoto, R.G. Nuzzo, L. Mahadevan, J.A. Lewis, Biomimetic 4D printing. Nat. Mater. 15, 413 (2016)

14. A. Bastola, M. Paudel, L. Li, Development of hybrid magnetorheological elastomers by 3D printing. Polymer 149, 213 (2018)

15. M. Balk, M. Behl, U. Nöchel, A. Lendlein, Architectured Shapememory hydrogels with switching segments based on oligo (e-caprolactone). MRS Adv. 1, 2011 (2016)

16. STRATASYS: the 3D Printing Solutions Company: polyjet simulated rubber material. https://www.stratasys.com/materials/. Accessed 29 Oct 2020.

17. A.K. Bastola, N. Rodriguez, M. Behl, P. Soffiatti, N.P. Rowe, A. Lendlein, Cactus-inspired design principles for soft robotics based on 3D printed hydrogel-elastomer systems. Mater. Des. 202, 109515 (2021) 